\section{Development of a simple computerized torsion test to quantify subjective ocular torsion}

YD Kim ${ }^{1}$ HK Yang ${ }^{1}$ and J-M Hwang
Department of

Ophthalmology, Seoul National University College of Medicine, Seoul National University Bundang Hospital, Seongnam, Korea

\section{Correspondence:} J-M Hwang, Department of Ophthalmology, Seoul

National University College of Medicine, Seoul National University Bundang Hospital, 166 Gumiro, Bundang-gu, Seongnam 463-707, Korea

Tel: +82 31787 7372;

Fax: +82 317874057 .

E-mail: hjm@snu.ac.kr

${ }^{1}$ These authors contributed equally to this work.

Received: 22 January 2017 Accepted in revised form: 24 March 2017

Published online:

16 June 2017

\begin{abstract}
Purpose The double Maddox-rod test (DMRT) and Lancaster red-green test (LRGT) are the most widely used tests worldwide to assess subjective ocular torsion. However, these tests require equipment and the quantified results of ocular torsion are only provided in rough values. Here we developed a novel computerized torsion test (CTT) for individual assessment of subjective ocular torsion and validated the reliability and accuracy of the test compared with those of the DMRT and LRGT.

Methods A total of 30 patients with cyclovertical strabismus and 30 controls were recruited. The CTT was designed using Microsoft Office PowerPoint. Subjects wore red-green filter spectacles and viewed gradually tilted red and cyan lines on an LCD monitor and pressed the keyboard to go through the slides, until both lines seemed parallel. All subjects underwent the CTT, DMRT, and LRGT. Intraclass correlation coefficients and Bland-Altman plots were analyzed to assess the acceptability of the CTT compared with that of the DMRT. Results Both the DMRT and CTT showed no significant test-retest differences in the strabismus and control groups. The DMRT and CTT results demonstrated an acceptable agreement. The reliability of the CTT was better than that of the DMRT. The LRGT showed low sensitivity for the detection of ocular torsion compared with the DMRT (40.0\%) and CTT (39.1\%).

Conclusion Our results suggest that the assessment of subjective ocular torsion using the CTT based on PowerPoint software is simple, reproducible, and accurate and can be applied in clinical practice.

Eye (2017) 31, 1562-1568; doi:10.1038/eye.2017.90; published online 16 June 2017
\end{abstract}

\section{Introduction}

Cyclotropia results from misalignment along the anteroposterior axis between the two eyes. ${ }^{1}$ Among the heterogeneous causes of cyclodeviation, torsional diplopia has been recognized in $13 \%$ patients with strabismus and cyclodeviation, and this cannot be corrected with prisms. $^{2}$ Therefore, the documentation and diagnosis of cyclodeviation is essential in the management of strabismus. ${ }^{3}$

Subjective ocular torsion could be measured using the double Maddox-rod test (DMRT), Lancaster red-green test (LRGT), unmounted double Bagolini lenses, the torsionometer and synoptophore. ${ }^{4-9}$ The DMRT is the most commonly used method for the quantitative measurement of cyclodeviation; however, it has some limitations. First, there is complete visual deprivation except for light coming through the Maddox-rods lenses during the test. ${ }^{7}$ Visual deprivation interferes with adaptation mechanisms, which may not reflect the physiological perception of cyclodeviation in ordinary situations in patients with strabismus., ${ }^{4,7}$ Second, the DMRT cannot detect small changes, because the intervals on trial frames are usually $5^{\circ} .9$ The LRGT offers the advantage of torsion measurement in the nine diagnostic positions of the gaze. ${ }^{4,5}$ However, it measures vertical and horizontal components in addition to the pure torsional misalignment, and large horizontal or vertical deviations interfere with the measurement of cyclodeviation. The quantification of cyclodeviation using the LRGT is also limited, and small amounts of torsion can be neglected by the examiner. The torsionometer developed by Georgievski is portable and easy to use for both patient and examiner. ${ }^{6}$ However, it can measure only up to $25^{\circ}$ of torsion. The Bagolini striated lens test also could measure cyclotropia under nearly 
normal binocular viewing conditions. ${ }^{7,8}$ Ruttum and von Noorden ${ }^{7}$ claimed that the torsion measured with the Bagolini lens are similar to those with the DMRT. However, Kraft et $a l^{8}$ reported that the DMRT was better than the Bagolini lens for the diagnosis of unilateral superior oblique palsy as well as differentiation of bilateral vs unilateral superior oblique palsy, thus no longer recommended the use of Bagolini lens for the evaluation of superior oblique palsy. Synoptophore measures ocular torsion; however, it is dissociative, less physiological, and requires special equipment. $^{10}$

In the present study, we developed a novel subjective torsion test designed using Microsoft Office PowerPoint for the individual assessment of subjective ocular torsion using red and green filters and validated the reliability and accuracy of the test compared with those of the DMRT and LRGT.

\section{Materials and methods}

\section{Study participants}

A total of 30 patients with cyclovertical strabismus and 30 controls without any strabismus were included in this retrospective study, which was performed in accordance with the Declaration of Helsinki and was approved by the institutional ethics committee of the Seoul National University Bundang Hospital. The required sample size of the patient group $(n=29)$ was calculated based on the assumption of an effect size of 0.7 (estimated difference of test results between two dependent means/SD), $\alpha$ error $=5 \%$ and power $=95 \%$ (G-power).

Cyclovertical strabismus was diagnosed on the basis of ocular motility evaluations and/or subjective image tilting. The exclusion criteria were as follows: impairment of perception or general motor function, impairment of color vision, and a best corrected visual acuity (BCVA) of $<20 / 40$ in either eye. Individuals with no history of torsional strabismus nor diplopia were voluntarily recruited as controls.

All subjects were examined under a uniform protocol including BCVA and assessment of ocular alignment in the seven cardinal positions of gaze as part of the routine clinical examination. All subjects underwent our newly developed computerized torsion test (CTT), DMRT, and LRGT in random order on the same day.

\section{Computerized torsion test}

The subjects wore red-green filter spectacles (common and commercially available red-green glasses that are usually used for the Worth-four-dot test or the LRGT), with the red filter over the right eye and the green filter over the left eye. ${ }^{11}$ Trained instructors monitored the subjects to ensure a straight head position throughout the examination. The examiner continued to progress through the slides until the subject reports that the lines are parallel. The CTT was designed using Microsoft Office PowerPoint 2007 (Microsoft, Redmond, WA, USA) and was administered to the subjects on a 27-inch liquid crystal display (LCD) monitor (Samsung Electronics Co., Ltd., Suwon, South Korea) at a working distance of $50 \mathrm{~cm}$. The CTT evaluated torsional cyclodeviation using two different colored lines: red and cyan. The cyan line was made with an equal proportion of blue and green. Cyan targets were used instead of green because only cyan was completely filtered using a green filter. The red line was invisible when viewed through the red filter, and the cyan line was invisible when viewed through the green filter. All the other colors appeared black; therefore, binocular viewing of the red and cyan lines through the red and green filters showed two dark lines, one in each eye (Figure 1). Every slide was constructed with five concentric circles, a red line and a cyan line horizontally passing through the circles (Figure 1). The concentric circles were used as a fixation target. Two parallel horizontal lines were demonstrated in the first slide: an upper red line and a lower cyan line. If the subject wearing red-green filter spectacles stated that the upper line (red line) was tilted, he/she was diagnosed with ocular torsion in the left eye. If the subject stated that the

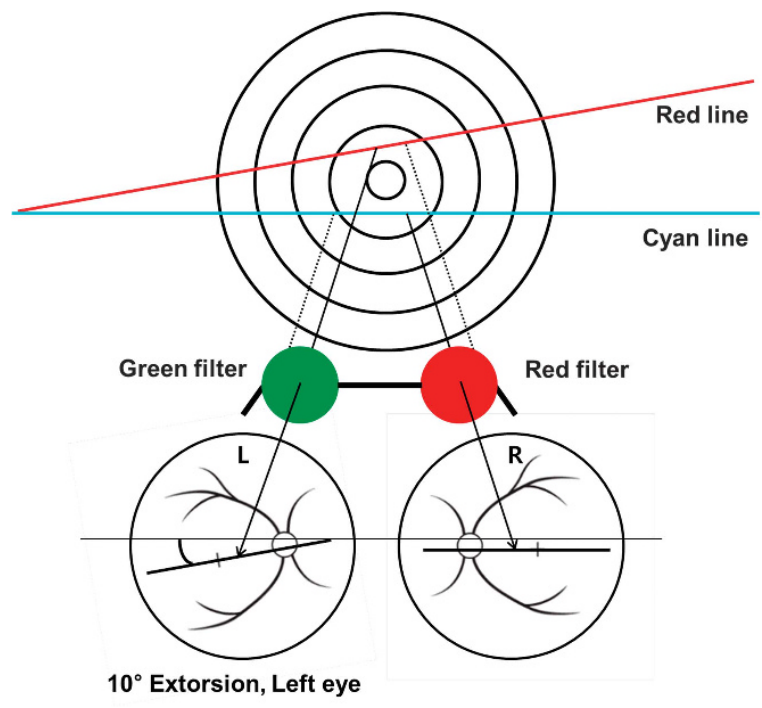

Figure 1 Conceptual image of the computerized torsion test (CTT). The red line is invisible through the red filter and the cyan line is invisible through the green filter. The filtered color is invisible to the subject, and the other colors are viewed as a black line. In this example, the upper line is only visible to the left eye and the lower line is only visible to the right eye. The subject is requested to press the right arrow key to go forward through the slide show until the two lines appear perfectly parallel. If the subject perceives the two lines as parallel in the above figure, he will be diagnosed with $10^{\circ}$ extorsion in the left eye. 
lower line (cyan line) was tilted, then he/she was diagnosed with torsion in the right eye.

If the subject noted that the upper red line was tilted (torsion in the left eye) in the first slide, the instructor pressed the right arrow key to go to the next slides, and the upper line (red line) was gradually slanted from $-30^{\circ}$ to $+30^{\circ}$ by $1^{\circ}$ intervals on each slide. If the subject noted the lower cyan line to be tilted (torsion in the right eye) in the first slide, then the instructor pressed the end key to go to the last slide and then pressed the left arrow key to perform the test with the upper line (cyan line) gradually slanted from $-30^{\circ}$ to $+30^{\circ}$ by $1^{\circ}$ interval on each slide (backward progression). In other words, there were two test sets for each situation (torsion in the left eye or right eye), respectively. Localization of torsion is not essential for the CTT, and some patients who did not perceive line tilting or perceived the tilting of both lines performed the test in sequence.

Figure 1 shows the conceptual image of the CTT. Two independent examiners (A and $\mathrm{B}$ ) repeated the test twice. Subjects showing intorsion or extorsion of $\geq 1^{\circ}$ were considered to have cyclodeviation. The obtained values were described on the slide note (eg, $10^{\circ}$ extorsion, left eye).

\section{Double maddox-rod test}

Red and white Maddox-rod lenses were respectively placed in a trial frame and applied in front of both eyes. The direction of the glass rods were marked on the margin of the Maddox-rod lenses and carefully aligned with the $90^{\circ}$ axis of the trial frame. To prevent unintentional head tilting, the examiners ensured that the horizontal position of the subject's eyes were leveled using the head and chin rest. The subject was instructed to look straight and fixate on an illuminator located $1 \mathrm{~m}$ away in the middle of both eyes. The images of light passed through the Maddox-rod lenses appear as red and white streaks on each eye.

Subjects were asked which colored line was slanted, then they rotated the lens of the tilted side until the streaks became perfectly parallel or overlapped. Two independent examiners performed the test twice and read to the nearest degree. Subjects showing intorsion or extorsion of $\geq 2^{\circ}$ were considered to have cyclodeviation.

\section{Lancaster red-green test}

The LRGT was performed using red-green filter spectacles and a head rest. Subjects denoted the light by a hand-held light source in nine diagnostic positions of gaze. Then, the examiner recorded the results using an electronic painting program. The presence of cyclodeviation was judged on the basis of the pictures created by the LRGT tester. Cyclodeviation was assessed in a binary scale as positive or negative by the LRGT. The sensitivity and specificity of the LRGT for detecting ocular torsion were determined in comparison with those of the DMRT and CTT.

\section{Data analysis}

The presence and direction of torsion by the DMRT, CTT and LRGT were defined and the absolute values of cyclodeviation by the DMRT and CTT were analyzed. One-way analysis of variances (ANOVA) was used to compare the four sets of DMRT and CTT data in the two groups. To analyze the test-retest reliability, intraclass correlation coefficients (ICCs; two-way mixed) were computed. A power value of $>0.80$ to detect an ICC was considered an acceptable reflection of reliability. Paired $t$-tests were used to assess differences between the DMRT and CTT values in the two groups. The Bland-Altman procedure was performed for each subject to check for dispersibility of the DMRT and CTT values. The sensitivity and the specificity of the DMRT and CTT for the detection of cyclovertical strabismus were determined by receiver operating characteristic (ROC) curves and areas under the receiver operating characteristic curves (AUCs). The sensitivity and specificity of the LRGT were analyzed and compared with those of the DMRT and CTT. The Mann-Whitney U test was used to evaluate differences in DMRT and CTT values between the LRGTpositive and LRGT-negative groups. Statistical analyses were performed using SPSS 21.0 Statistical Analysis Software (IBM, New York, NY, USA). Bland-Altman analysis was conducted using MedCalc 13.2 (MedCalc software, Ostend, Belgium).

\section{Results}

\section{Subject characteristics}

Thirty patients with cyclovertical strabismus (16 men, 14 women; age range, 5-70 years; mean age, $46.8 \pm 18.4$ years) and 30 controls (10 men, 20 women; age range, $31-74$ years; mean age, $46.7 \pm 13.0$ years) participated in this study.

The strabismus group comprised patients with superior oblique palsy ( $n=21,70.0 \%$ ), thyroid-associated orbitopathy $(n=2,6.7 \%)$, oblique muscle overaction $(n=2,6.7 \%)$, ocular myasthenia gravis $(n=1,3.3 \%)$, hypertropia $(n=1,3.3 \%)$, sagging eye syndrome $(n=1,3.3 \%)$, partial third nerve palsy $(n=1,3.3 \%)$, and diplopia of unspecified strabismus $(n=1,3.3 \%$; Table 1). 
Table 1 Clinical diagnosis and direction of ocular torsion in patients with cyclovertical strabismus and healthy controls

\begin{tabular}{lccccc}
\hline & No. of cases (\%) & Extorsion $($ DMRT) & ${\text { Intorsion }(D M R T)^{\mathrm{a}}}^{\mathrm{a}}$ & ${\text { Extorsion }(C T T)^{\mathrm{a}}}^{\text {Intorsion }_{(\mathrm{CTT})}}$ \\
\hline Control & $30(100 \%)$ & 0 & 0 & 0 & $1(3.3 \%)$ \\
Strabismus & $30(100 \%)$ & $19(63.3 \%)$ & $2(6.7 \%)$ & $22(73.3 \%)$ & $2(6.7 \%)$ \\
$\quad$ Superior oblique palsy & $21(70.0 \%)$ & $15(50.0 \%)$ & 0 & $17(56.7 \%)$ & 0 \\
Thyroid-associated orbitopathy & $2(6.7 \%)$ & $1(3.3 \%)$ & 0 & $1(3.3 \%)$ & 0 \\
Oblique muscle overaction & $2(6.7 \%)$ & $1(3.3 \%)$ & $1(3.3 \%)$ & $1(3.3 \%)$ & $1(3.3 \%)$ \\
Ocular myasthenia gravis & $1(3.3 \%)$ & 0 & $1(3.3 \%)$ & 0 & $1(3.3 \%)$ \\
Hypertropia & $1(3.3 \%)$ & 0 & 0 & $1(3.3 \%)$ & 0 \\
Sagging eye syndrome & $1(3.3 \%)$ & $1(3.3 \%)$ & 0 & 0 & 0 \\
Partial CN3 palsy & $1(3.3 \%)$ & 0 & 0 & $1(3.3 \%)$ & 0 \\
Unspecified diplopia & $1(3.3 \%)$ & $1(3.3 \%)$ & & 0 \\
\hline
\end{tabular}

Abbreviations: CTT, computerized torsion test; DMRT, double Maddox-rod test.

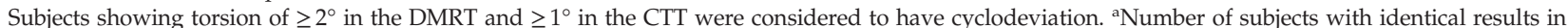
three or more test sets.

Table 2 Double Maddox-rod test and computerized torsion test results in the strabismus and control groups

\begin{tabular}{|c|c|c|c|c|c|}
\hline Method & $A-1$ & $A-2$ & $B-1$ & $B-2$ & $P$-value ${ }^{\mathrm{a}}$ \\
\hline \multicolumn{6}{|l|}{ Strabismus } \\
\hline $\operatorname{DMRT}\left({ }^{\circ}\right)$ & $5.37 \pm 3.69$ & $4.90 \pm 3.72$ & $3.80 \pm 3.33$ & $3.47 \pm 2.92$ & 0.11 \\
\hline CTT $\left({ }^{\circ}\right)$ & $3.40 \pm 3.29$ & $3.17 \pm 3.00$ & $2.67 \pm 2.52$ & $2.63 \pm 2.55$ & 0.67 \\
\hline \multicolumn{6}{|l|}{ Controls } \\
\hline $\operatorname{DMRT}\left({ }^{\circ}\right)$ & $0.37 \pm 1.06$ & $0.17 \pm 0.46$ & $0.07 \pm 0.25$ & $0.03 \pm 0.18$ & 0.14 \\
\hline $\mathrm{CTT}\left(^{\circ}\right)$ & $0.13 \pm 0.35$ & $0.13 \pm 0.35$ & $0.10 \pm 0.31$ & $0.07 \pm 0.25$ & 0.82 \\
\hline
\end{tabular}

Abbreviations: CTT, computerized torsion test; DMRT, double Maddox-rod test.

A-1: The first test set of the first examiner.

A-2: The second test set of the first examiner.

B-1: The first test set of the second examiner.

B-2: The second test set of the second examiner.

Values are expressed as means \pm standard deviations. ${ }^{a}$ Values were compared using one-way analysis of variance.

\section{Test-retest and intermethod reliability}

ANOVA revealed no significant differences among the four sets of DMRT and CTT data in the strabismus and control groups (Table 2).

Reliability of the CTT and the DMRT were obtained with ICC in both groups. An ICC of $>0.80$ was considered as acceptable. In the strabismus group, the CTT showed good test-retest reliability (ICC $=0.96 ; 95 \%$ confidence interval (CI), 0.93-0.98; $P<0.01$ ), while the DMRT showed acceptable test-retest reliability (ICC $=0.80 ; 95 \%$ CI, $0.65-0.90 ; P<0.01$ ). The ICC value for all the DMRT and CTT test sets was $0.91(95 \% \mathrm{CI}$, $0.85-0.95 ; P<0.01)$.

In the control group, the CTT showed good test-retest reliability (ICC $=0.81 ; 95 \% \mathrm{CI}, 0.67-0.90 ; P<0.01$ ), whereas the DMRT showed poor test-retest reliability (ICC $=0.32 ; 95 \% \mathrm{CI},-0.19-0.64 ; P=0.09)$. The ICC value for all DMRT and CTT test sets was 0.52 (95\% CI, 0.21-0.74; $P<0.01$ ).

Paired $t$-tests revealed significant differences between the DMRT and CTT values in the strabismus group $(P<0.001)$, but not in the control group $(P=0.42)$. The difference between the mean DMRT and CTT values was $1.42 \pm 2.06^{\circ}$ (DMRT, $4.38 \pm 3.47^{\circ}$; CTT, $2.97 \pm 2.84^{\circ}$ ) in the strabismus group and $0.50 \pm 0.67^{\circ}$ (DMRT, $0.16 \pm 0.61^{\circ}$; CTT, $0.11 \pm 0.31^{\circ}$ ) in the control group.

In Bland-Altman analysis, the mean difference between the DMRT and CTT values was $1.4 \pm 3.3^{\circ}$, however, the $95 \% \mathrm{CI}$ showed a wide range of $+7.9^{\circ}$ to $-5.1^{\circ}$. There was a trend of irregular dispersion; the DMRT values were smaller than the CTT values when the mean value for both tests was $<2^{\circ}$, and larger than the CTT values when the mean value for both tests was between $2^{\circ}$ and $5^{\circ}$ (Figure 2).

\section{ROC curves for the sensitivity and specificity of the DMRT and CTT for the detection of cyclovertical strabismus}

The sensitivity and specificity of the DMRT and CTT for the detection of cyclovertical strabismus were determined by ROC curves and AUCs. The AUC was the largest for a cut-off value of $1.8^{\circ}$ for the DMRT (AUC $=0.90$; sensitivity, $83.3 \%$; specificity, $100 \%$ ) and $0.38^{\circ}$ for the CTT 


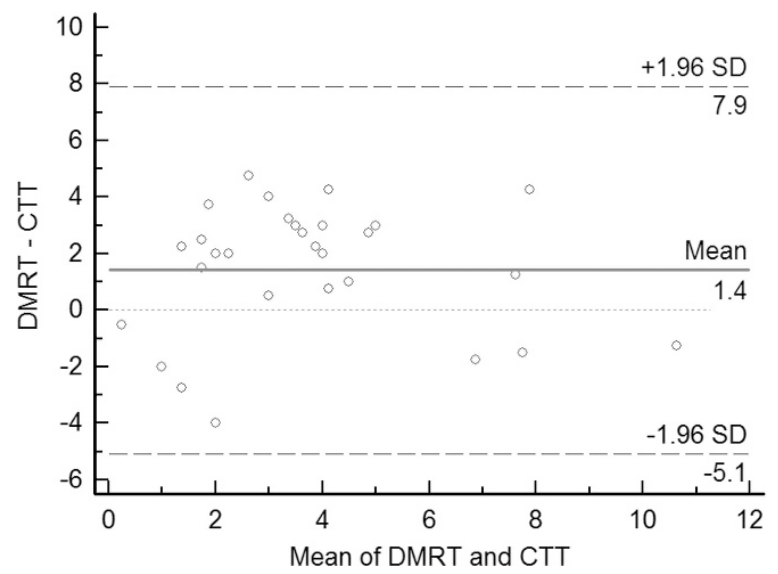

Figure 2 Bland-Altman plots comparing the degrees of ocular torsion measured by the double Maddox-rod test (DMRT) and computerized torsion test (CTT) relative to the mean value for both tests. The CTT demonstrates good agreement with the DMRT. However, there is a trend of irregular dispersion. The DMRT values are smaller than the CTT values when the mean value is $<2^{\circ}$, and larger than the CTT values when the mean value is between $2^{\circ}$ and $5^{\circ}$.

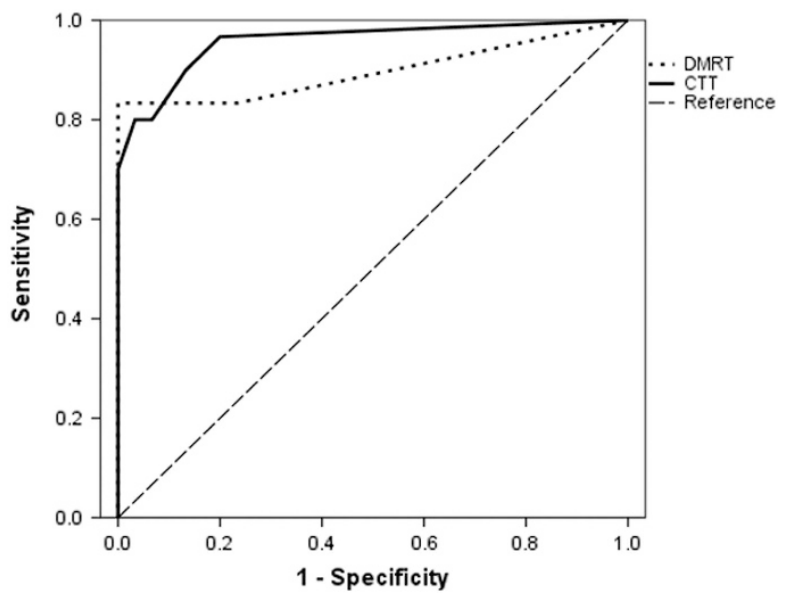

Figure 3 Receiver operating characteristic (ROC) curves for the sensitivity and specificity of the double Maddox-rod test (DMRT) and computerized torsion test (CTT) for the detection of cyclovertical strabismus. The area under the curve (AUC) is the largest for a cut-off value of $1.8^{\circ}$ for the DMRT (AUC $=0.90$; sensitivity, $83.3 \%$; specificity, $100 \%$ ) and $0.38^{\circ}$ for the CTT (AUC $=0.96$; sensitivity, $90.0 \%$; specificity, $86.7 \%$ ).

(AUC $=0.96$; sensitivity, 90.0\%; specificity, $86.7 \%$; Figure 3).

\section{Comparison with the LRGT}

Twenty-eight patients with strabismus performed the LRGT. Of these, nine showed positive cyclodeviation and 19 showed negative cyclodeviation. Eight of the nine
LRGT-positive patients also showed subjective ocular torsion in both the DMRT and CTT performed simultaneously. One patient only showed positive cyclodeviation in the CTT (mean, $4^{\circ}$ ).

The mean DMRT and CTT values were significantly different between the LRGT-positive and LRGT-negative groups $(P<0.01$; Mann-Whitney $U$ test). The mean DMRT values were $5.9 \pm 3.9$ (range, $0-15$ ) in the LRGT-positive group and $3.8 \pm 3.1$ (range, $0-10$ ) in the LRGT-negative group. The mean CTT values were $4.7 \pm 3.4$ (range, $1-12$ ) in the LRGT-positive group and $2.3 \pm 3.1$ (range, $0-10$ ) in the LRGT-negative group. The LRGT-positive group showed significantly larger mean values compared to LRGT-negative group in both DMRT $(P<0.01)$ and CTT $(P<0.01)$. The overall sensitivity of the LRGT was $32.1 \%$. Compared with the DMRT as a gold standard, the sensitivity of the LRGT was $40.0 \%$ and specificity was $87.5 \%$. Compared with the CTT as a gold standard, the values were $39.1 \%$ and $100 \%$, respectively.

\section{Discussion}

In the present study, we developed a new computer based test for the measurement of subjective ocular torsion and evaluated its reliability compared with the DMRT and LRGT. We found that the CTT had better reproducibility and higher sensitivity than the DMRT.

The main advantages of the CTT over the DMRT are its higher reliability and easy operation. First, the CTT can measure subtle differences by an interval of $1^{\circ}$. The DMRT cannot detect such small changes, because the interval on trial frames is $5^{\circ}$, which does not allow fine measurements. ${ }^{9}$ Interpretation of the DMRT is also examiner-dependent, which results in low reproducibility. Poor fitting between the lens and frame also results in clumsy interpretation of the results; furthermore, fine adjustment of rotation with the Maddox-rods is somewhat difficult for young and aged patients. These limitations make the DMRT a somewhat unreliable test. ${ }^{9}$ On the other hand, a simple control of rotation in pre-fixed intervals using the keyboard is much easier than adjusting the Maddox-rod with fingers, particularly if the angle of torsion is small. Second, the CTT can be performed by following simple manuals without a background knowledge, and the result can be directly reported as shown in the slides, without additional interpretation. On the contrary, examiners must understand the test mechanism of the DMRT for accurate interpretation of the results. The DMRT is sometimes difficult to understand for both the examiner and patient with strabismus. Special care to avoid tilting the trial frames during the study is necessary and a 
vertical prism may be added to separate the images to facilitate identification. ${ }^{12}$ Moreover, the CTT theoretically allows testing in variable positions of gaze. Although the DMRT has been tried in variable positions of gaze, only patients who could follow the instructions to rotate the Maddox-lens were capable of doing the test. Poor fixation during the DMRT due to visual suppression by the striated lens decreases the reliability of the effect of positional changes. The LRGT works in nine diagnostic positions of gaze; however, its qualitative or semiquantitative characteristic is a major limitation. In the present study, the CTT was only performed in the primary position, although it could be performed in different positions to provide interesting and valid outcomes. For example, a decrease in ocular torsion from the upright position to the supine position may indicate skew deviation, ${ }^{13}$ and the CTT, which is implemented using portable devices, may be able to differentiate between trochlear nerve palsy and skew deviation.

Another explanation for the difference between the DMRT and CTT is the complete dissociating characteristics of the former. Complete deprivation of visual perception with striated lens may interfere with adaptation mechanisms; consequently, a positive DMRT may be found in an asymptomatic patient with cyclotropia. ${ }^{3}$ On the other hand, the CTT is performed with the visual environment maintained in a near normal condition, except the background color of the lens. Moreover, the eccentric circles in the center allow fusion of vertical or horizontal strabismus. Therefore, only the torsional component of diplopia can be measured by the CTT, and this is a powerful advantage of this test.

In the Bland-Altman plots, the CTT demonstrated good agreement with the DMRT. However, there was a trend of irregular dispersion. The DMRT values were smaller than the CTT values when the mean values for both tests were $<2^{\circ}$, and larger than the CTT values when the mean value was between $2^{\circ}$ and $5^{\circ}$. These differences cannot be ignored and can be amplified in larger ranges of cyclodeviation. Considering that the CTT is more accurate than the DMRT, this is probably another limitation of the DMRT.

The LRGT showed poor sensitivity for the detection of subjective ocular torsion in the present study. The LRGT is advantageous for testing paralytic strabismus by alternate fixation of each eye. However, subjective recording of the outcome results in a failure to detect subtle cyclodeviation, which is a major limitation of the LRGT. Moreover, cyclodeviation can also be masked by large horizontal or vertical deviations. Further studies are required to demonstrate changes in subjective torsion measured by the CTT in different cardinal positions.
A computerized LRGT was previously developed on a 40 -inch monitor operated by a joystick. ${ }^{14}$ This measured the horizontal, vertical, and torsional deviations in numerical values and showed good agreement with the LRGT. The computerized LRGT is advantageous for quantification; however, it is not commercially available and requires special equipment.

Commercial binocular torsion tests (i-Chart and Test Chart XPert 3Di) supplied with multiple vision tests are also available. ${ }^{15,16}$ The torsion tests included in these softwares also use cyan and red lines, and their principles are similar to those of the CTT. However, they have some limitations. First, both require expensive equipment. Second, because there were no fixation targets, patients with large horizontal or vertical deviations may not accurately match the two lines.

The optimal colors of red and cyan in our study were determined by trial and error. Actually, the three subtractive primary colors are magenta, cyan, and yellow. Interestingly, magenta and red expressed by display devices appear to have identical characteristics through the red filter, while cyan expressed on the LCD monitor has identical characteristics with the natural green color. A previous study used blue targets and blue filters, because green targets were not efficiently filtered on the LCD monitor. ${ }^{14}$ Because green filters are practically used in ophthalmology clinics, cyan targets are much more useful. The size of the monitor is not important, as long as it expresses undistorted images.

Our study has some limitations. First, the patients with strabismus had various disorders, which could have resulted in variable test outcomes. Second, the age range of participants was wide (5-70) which may have affected the reliability of results in very young and aged patients. However, the age distributions in both groups were not significantly difference which does not change our qualitative conclusions. Lastly, the LRGT was assessed in a binary scale and not in a quantified measurement as the DMRT or the CTT. This was because LRGT is practically used to find the presence of torsion and its direction, and not in a quantified manner.

Furthermore, the torsional angle may be erroneously copied by the examiner while drawing the results of the LRGT.

In conclusion, we developed a simple and efficient tool for the measurement of subjective ocular torsion using a common presentation software. We validated the reliability of the CTT and found excellent repeatability and accuracy of the results. The CTT may be used as a primary tool for the evaluation of cyclodeviation, with better reproducibility and higher sensitivity than the DMRT. 


\section{Summary}

What was known before

- Subjective ocular torsion could be measured using the double Maddox-rod test (DMRT), Lancaster red-green test (LRGT), and synoptophore.

- The DMRT is the most commonly used method for the quantitative measurement of cyclodeviation; however, it has some limitations.

- First, there is complete visual deprivation except for light coming through the Maddox-rod lenses during the test.

- Visual deprivation interferes with adaptation mechanisms, which may not reflect the physiological perception of cyclodeviation in ordinary situations in patients with strabismus.

- Second, the DMRT cannot detect small changes, because the intervals on trial frames are usually $5^{\circ}$.

- The LRGT offers the advantage of torsion measurement in the nine diagnostic positions of gaze.

- However, it measures vertical and horizontal components in addition to the pure torsional misalignment, and large horizontal or vertical deviations interfere with the measurement of cyclodeviation.

- The quantification of cyclodeviation using the LRGT is also limited, and small amounts of torsion can be neglected by the examiner.

- Synoptophore measures ocular torsion; however, it is dissociative, less physiological, and requires special equipment.

What this study adds

- In the present study, we developed a novel subjective torsion test designed using Microsoft Office PowerPoint for the individual assessment of subjective ocular torsion using red and green filters and validated the reliability and accuracy of the test compared with those of the DMRT and LRGT.

\section{Conflict of interest}

The authors declare no conflict of interest.

\section{References}

1 von Noorden GK, Campos EC. Binocular Vision and Ocular Motility: Theory and Management of Strabismus, 6th edn. Mosby Inc.: Philadelphia, USA, 2001, p 389.

2 Woo SJ, Seo JM, Hwang JM. Clinical characteristics of cyclodeviation. Eye (Lond) 2005; 19: 873-878.

3 von Noorden GK. Clinical and theoretical aspects of cyclotropia. J Pediatr Ophthalmol Strabismus 1984; 21: 126-132.

4 Lemos J, Eggenberger E. Clinical utility and assessment of cyclodeviation. Curr Opin Ophthalmol 2013; 24: 558-565.

5 Klainguti G, Strickler J, Chamero J. Comparison of five methods for subjective assessment of ocular cyclodeviation. Klin Monbl Augenheilkd 1992; 200: 409-413.

6 Georgievski Z. A new test for the measurement of torsion. J Pediatr Ophthalmol Strabismus 1996; 33: 63-64.

7 Ruttum M, von Noorden GK. The Bagolini striated lens test for cyclotropia. Doc Ophthalmol 1984; 58: 131-139.

8 Kraft SP, O'Reilly C, Quigley PL, Allan K, Eustis HS. Cyclotorsion in unilateral and bilateral superior oblique paresis. J Pediatr Ophthalmol Strabismus 1993; 30: 361-367.

9 Trobe JD. Cyclodeviation in acquired vertical strabismus. Arch Ophthalmol 1984; 102: 717-720.

10 Sharma P, Thanikachalam S, Kedar S, Bhola R. Evaluation of subjective and objective cyclodeviation following oblique muscle weakening procedures. Indian J Ophthalmol 2008; 56: 39-43.

11 Hwang JM, Guyton DL. The Lancaster red-green test before and after occlusion in the evaluation of incomitant strabismus. J AAPOS 1999; 3: 151-156.

12 von Noorden GK, Campos EC. Binocular Vision and Ocular Motility: Theory and Management of Strabismus 6th edn. Mosby Inc.: Philadelphia, PA, USA, 2001, pp 194-195.

13 Parulekar MV, Dai S, Buncic JR, Wong AMF. Head positiondependent changes in ocular torsion and vertical misalignment in skew deviation. Arch Ophthalmol 2008; 126: 899-905.

14 Awadein A. A computerized version of the Lancaster redgreen test. J AAPOS 2013; 17: 197-202.

15 No Authors. i-Chart The iMac vision test: a complete multilanguage electronic vision test system. Available at: http:/ /www.imac-visiontest.com/i-chart. Accessed 4 February 2016.

16 No Authors. Thomson Software Solutions: Test Chart 2016. Available at: http:/ /www.thomson-software-solutions. com/test-chart-xpert-3di. Accessed 4 February 2016. 